\title{
Dhouib-Matrix-TSP1 Method to Optimize Octagonal Fuzzy Travelling Salesman Problem Using $\alpha$-Cut Technique
}

\author{
(Dhouib-Matrix-TSP1 for Octagonal Fuzzy TSP)
}

\author{
Mariem Miledi \\ Department of Quality Management, \\ Higher Institute of Industrial \\ Management, \\ University of Sfax, \\ Tunisia
}

\author{
Souhail Dhouib \\ Department of Industrial Management, \\ Higher Institute of Industrial \\ Management, \\ University of Sfax, Tunisia \\ Email: souhail.dhouib [AT] gmail.com
}

\author{
Taicir Loukil \\ Department of Quantitative Method, \\ Faculty of Management and Economic \\ Sciences, \\ University of Sfax, \\ Tunisia
}

\begin{abstract}
This paper proposes the optimization of the fuzzy travel salesman problem by using the $\alpha$-Cut technique as a ranking function and the Dhouib-Matrix-TSP1 as an approximation method. This method is enhanced by the standard deviation metric and obtains a minimal tour in fuzzy environment where all parameters are octagonal fuzzy numbers.
\end{abstract}

Fuzzy numbers are converted into a crisp number thanks to the ranking function $\alpha$-Cut. The proposed approach in details is discussed and illustrated by a numerical example. This method helps in designing successfully the tour to a salesman on navigation through the distance matrix so that it minimizes the total fuzzy distance.

Keywords-component; Optimization; Dhouib-Matrix-TSP1; Fuzzy Numbers; Travelling Salesman Problem; Approximation Method; Ranking Function; $\alpha-C u t$ Technique

\section{INTRODUCTION}

In order to optimize the Travelling Salesman Problem (TSP) in polynomial computational time, a new approximation method named Dhouib-Matrix-TSP1 is designed and developed by Dhouib in $[1,2,3]$. The main role of this proposed method is to guide the navigation through the matrix distance in order to construct the minimal tour. In fact, in this paper, we will enhance the first phase of the Dhouib-MatrixTSP1 method by the insertion of the standard deviation metric instead of the minimization one. Then, we will apply the Dhouib-Matrix-TSP1 to solve the octagonal fuzzy TSP.

This paper is organized as follows. Firstly, in section 2, we recall the fuzzy TSP concepts. Then, in section 3, we present the Dhouib-Matrix-TSP1 method. In section 4, we discuss a detailed numerical application. Finally, in section 5, we give a conclusion.

\section{The FuZZY TRAVELLING SALESMAN PROBLEM}

The fuzzy set number represents the mathematical formulation of the impreciseness in the real-world which was introduced firstly by Zadeh [4, 5]. Fuzzy set theory is widely used in the Travel Salesman Problem where information (cost, time or distance) is not certain.

[6] uses a memetic random key algorithm for the balanced travelling salesman problem. [7] proves a discrete bat algorithm based on Lévy flights for Euclidean travelling salesman problem. [8] exploits a variable neighborhood search algorithm for solving the cost-balanced travelling salesman problem. [9] presents an Ant Colony Optimization metaheuristic to optimize the dynamic travelling salesman problem for a real-life case study. [10] uses a centroid ranking technique to optimize the fuzzy transportation problem. [11] uses the Artificial Bee Algorithm to generate the efficient routes for multi-objective TSP with uncertain variables on the arcs. [12] generates the Pareto optimal solutions for the travelling salesman problem minimizing the cost and time in a fuzzy environment. [13] presents a particle swarm optimization metaheuristic to optimize the travelling salesman problems with fuzzy means clustering. [14] develops a new method to optimize the fuzzy multi-objective transportation problem. [15] develops a balanced fuzzy particle swarm optimization algorithm for solving travelling salesman problem. [16] develops a genetic algorithm to solve an asymmetric travelling salesman problem where the distance is presented by an interval of values.

A fuzzy set is categorized by a membership function mapping element of a space in the interval [0,1]. The octagonal fuzzy number is denoted as $\left(a_{1}, a_{2}, a_{3}, a_{4}, a_{5}, a_{6}, a_{7}\right.$, $\left.a_{8}\right)$ and its membership function is given in (1). 


$$
\mu_{\lambda}(x) \begin{cases}0, & x<a_{1} \\ k\left(\frac{x-a_{1}}{a_{2}-a_{1}}\right) & a_{1} \leq x \leq a_{2} \\ k & a_{2} \leq x \leq a_{3} \\ k+(1-k)\left(\frac{x-a_{3}}{a_{4}-a_{3}}\right) & a_{3} \leq x \leq a_{4} \\ k\left(\frac{x-a_{1}}{a_{2}-a_{1}}\right) & a_{4} \leq x \leq a_{5} \\ k+(1-k)\left(\frac{a_{6}-x}{a_{6}-a_{5}}\right) & a_{5} \leq x \leq a_{6} \\ k & a_{6} \leq x \leq a_{7} \\ k\left(\frac{a_{8}-x}{a_{8}-a_{7}}\right) & a_{7} \leq x \leq a_{8} \\ 0 & x \geq a_{8} \quad \text { Where } 0<k<1\end{cases}
$$

Figure 1. depicts the graphical representation of an octagonal fuzzy numbers.

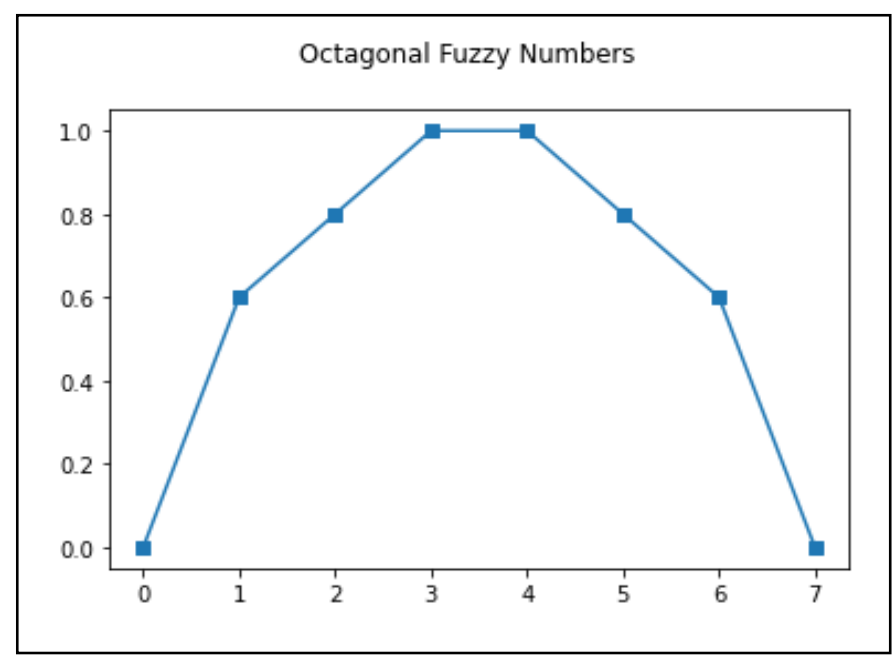

Figure 1. Graphical representation of an octagonal fuzzy numbers

\section{The PROPOSED Methodology: THE DHOUIB-MATRIX- TSP1}

Recently, [1] designed a new approximation method named Dhouib-Matrix-TSP1 to solve the Travelling Salesman Problem in polynomial computational time. In this paper, we will enhance the first phase of this new method with a modification in the system of measurement: we will use the standard deviation metric instead of the minimization metric as in [1]. The Dhouib-Matrix-TSP1 is composed of a very simple four phases repeated $n$ iterations where $n$ is the number of cities.

The four simple phases are described as follows:
- Phase 1: Compute the standard deviation for each row and write it on the right-hand side of the matrix as a list. Then, select the smallest element in this list, this element will specify the corresponding row and search its smallest element: this is the two first cities to be inserted in the proposed route Route-cities $\{x, y\}$ and discard their columns.

- $\quad$ Phase 2: Find the smallest element for $x$ and $y$ rows and choose the smallest distance, let it be the city $z$.

- $\quad$ Phase 3: Add the last selected city $z$ in the Route-cities at right if $\beta$ it_comes fropq) city $y_{(\text {Pr }}$ at left if it comes from city $x$. Next, discard the column of the last selected city and return to Phase 2 only if there are cities not in the list: Route-cities, otherwise start Phase 4.

- $\quad$ Phase 4: Modify the generated route Route-cities to be a tour by transforming the position of the first element to the end (one by one) until the starting city will be at the first position. To finish this tour, add the starting city as the last position.

\section{NUMERICAL APPLICATION TO THE OCTAGONAL FUZZY TRAVEL SALESMAN PROBLEM}

Consider the fuzzy travelling salesman problem in Figure 2, to solve it as a minimization of the total distance (five cities) using Dhouib-Matrix-TSP1 method.

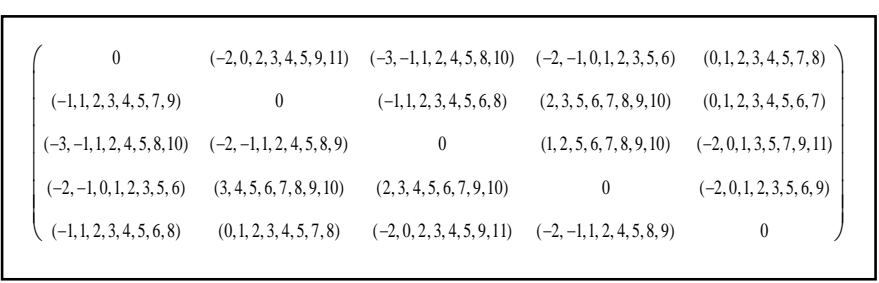

Figure 2. Fuzzy distance matrix

To solve this problem using Dhouib-Matrix-TSP1 method with some modification in the standard deviation metric, we need, first, to apply a flexible ranking function which satisfies the decision maker preference with compensation, linearity and additive properties. In this paper, we will use the robust $\alpha$ Cut index technique which is defined by: $R(a)=\int_{0}^{1}(0.5)\left(\alpha_{\alpha}^{L}, \alpha_{\alpha}^{U}\right) d \alpha$

Where

$\left(\alpha_{\alpha}^{L}, \alpha_{\alpha}^{U}\right)=[\{(b-a) \alpha+a, d-(d-c) \alpha\},\{(f-e) \alpha+e, h-(h-g) \alpha\}]$

Then, we use the $\alpha$-Cut technique to convert the octagonal fuzzy number to a crisp number as follows:

$R(-2,0,2,3,4,5,9,11)=\int_{0}^{1}(0.5)(\{2 \alpha-2,3-\alpha\},\{\alpha+4,11-2 \alpha\}) d \alpha=8$

Similarly, we convert the other elements of the matrix (see TABLE 1)

TABLE I. CONVERTING THE OCTAGONAL NUMBERS 


$$
\begin{array}{cc}
R(-3,-1,1,2,4,5,8,10)=6.5 & R(-2,-1,0,1,2,3,5,6)=3.5 \\
R(0,1,2,3,4,5,7,8)=7.5 & R(-1,1,2,3,4,5,7,9)=7.5 \\
R(-1,1,2,3,4,5,6,8)=7 & R(2,3,5,6,7,8,9,10)=12.5 \\
R(0,1,2,3,4,5,6,7)=7 & R(-3,-1,1,2,4,5,8,10)=6.5 \\
R(-2,-1,1,2,4,5,8,9)=6.5 & R(1,2,5,6,7,8,9,10)=12 \\
R(-2,0,1,3,5,7,9,11)=8.5 & R(-2,-1,0,1,2,3,5,6)=3.5 \\
R(3,4,5,6,7,8,9,10)=13 & R(2,3,4,5,6,7,9,10)=11.5 \\
R(-2,0,1,2,3,5,6,9)=6 & R(-1,1,2,3,4,5,6,8)=7 \\
R(0,1,2,3,4,5,7,8)=7.5 & R(-2,0,2,3,4,5,9,11)=8 \\
R(-2,-1,1,2,4,5,8,9)=6.5 & \\
\hline
\end{array}
$$

Thus, the table of fuzzy distances can be reduced to crisp numbers as presented in Figure 3.

$\left(\begin{array}{ccccc|}0 & 8 & 6.5 & 3.5 & 7.5 \\ 7.5 & 0 & 7 & 12.5 & 7 \\ 6.5 & 6.5 & 0 & 12 & 8.5 \\ 3.5 & 13 & 11.5 & 0 & 6 \\ 7 & 7.5 & 8 & 6.5 & 0\end{array}\right)$

Figure 3. Crisp distance matrix

Next, we can start the Dhouib-Matrix-TSP1 method, which just needs four iterations $(n=4)$ with four phases in each one to find the optimal solution which is equal to 30 .

Iteration1: Compute the standard deviation (whereas, in [1] Dhouib use the minimization metric) for each row and write it on the right-hand side of the matrix (see Figure 4).

\begin{tabular}{|ccccc|c|}
\hline 0 & 8 & 6.5 & 3.5 & 7.5 & 2.98 \\
7.5 & 0 & 7 & 12.5 & 7 & 3.98 \\
6.5 & 6.5 & 0 & 12 & 8.5 & 3.91 \\
3.5 & 13 & 11.5 & 0 & 6 & 4.87 \\
7 & 7.5 & 8 & 6.5 & 0 & 2.94 \\
\hline
\end{tabular}

Figure 4. Compute the standard deviation for each row

\begin{tabular}{|c|c|c|c|c|c|}
\hline 0 & 8 & 6.5 & 3.5 & 7.5 & \\
\hline 7.5 & 0 & 7 & 12.5 & 7 & \\
\hline 6.5 & 6.5 & 0 & 12 & 8.5 & . 5-4-1-3 \\
\hline 3.5 & 13 & 11.5 & 0 & 6 & Discard column 3 \\
\hline 7 & 7.5 & 8 & 6.5 & 0 & \\
\hline
\end{tabular}

The smallest element in this list is 2.94 in row 5. Then, search in row 5 the smallest element which is 6.5 in position $d_{54}$, insert cities 5 and 4 in Route-cities $\{5,4\}$ and discard the columns of cities 5 and 4 (see Figure 5).

$\left(\begin{array}{ccccc}0 & 8 & 6.5 & 3.5 & 7.5 \\ 7.5 & 0 & 7 & 12.5 & 7 \\ 6.5 & 6.5 & 0 & 12 & 8.5 \\ 3.5 & 13 & 11.5 & 0 & 6 \\ 7 & 7.5 & 8 & 6.5 & 0\end{array}\right)$ Discard columns 5 et 4

Figure 5. Select cities 4 and 5 and discard their columns
Iterations 2: Find the smallest element of row number 5 which is 7 and row number 4 which is 3.5 . Then, choose the smallest distance, the 3,5 in the position $d_{41}$, insert the city 1 to the Route-cities $\{5,4,1\}$ and discard the column of city 1 (see Figure 6).

$\left.\begin{array}{|ccccc}0 & 8 & 6.5 & 3.5 & 7.5 \\ 7.5 & 0 & 7 & 12.5 & 7 \\ 6.5 & 6.5 & 0 & 12 & 8.5 \\ 03.5 & 13 & 11.5 & 0 & 6 \\ & 7.5 & 8 & 6.5 & 0\end{array}\right)$ Discard column 1

Figure 6. Select city 1 and discard its column

Iterations 3: Find the smallest element for row number 5 which is 7.5 and row number 1 which is 6.5 . Then, choose the smallest distance, the 6,5 in the position $d_{13}$, insert the city 3 to the Route-cities $\{5,4,1,3\}$ and discard the column of city 3 (see Figure 7).

Figure 7. Select city 3 and discard its column

Iterations 4: Find the smallest element for row number 5 which is 7.5 and row number 3 which is 6.5 . Then, choose the smallest distance which is 6.5 in the position $d_{32}$, insert the city 2 to the Route-cities $\{5,4,1,3,2\}$ and discard the column of city 2 (see Figure 8).

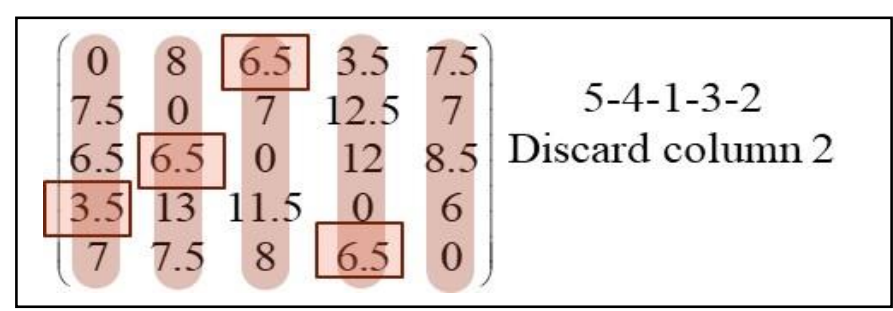

Figure 8 . Select city 2 and discard its column

Finally, to generate a tour from the route Route-cities $\{5,4$, $1,3,2\}$, translate the position of city 5 to the end $\{4,1,3,2,5\}$. Repeat the same thing to city number $4\{1,3,2,5,4\}$ and finally add the starting city to the last position $\{1,3,2,5,4,1\}$. So, our method finds the optimal solution (30) only in 4 simple iterations.

\section{CONCLUSION}

In this paper, the Dhouib-Matrix-TSP1 method is presented to solve octagonal fuzzy Travel Salesman Problem (TSP). Furthermore, this method is improved by the standard 
deviation metric for finding the optimal tour of a fuzzy TSP. The Dhouib-Matrix-TSP1 is simple, easy to understand and solves the problem in only $n$ iterations. The fuzzy numbers are reduced through the robust $\alpha$-Cut ranking function and the optimal solution for the considered example is obtained. Further research will apply this Dhouib-Matrix-TSP1 heuristic method on TSP benchmark instances.

\section{REFERENCES}

[1] S. Dhouib, "A New Column-Row Method for Traveling Salesman Problem: The Dhouib-Matrix-TSP1", International Journal of Recent Engineering Science, 2021, vol. 8, Issue 1, pp.6-10.

[2] S. Dhouib, "Stochastic Column-Row Method for Travelling Salesman Problem: the Dhouib-Matrix-TSP2", International Journal of Engineering Research \& Technology, 2021, vol. 10, Issue 3, pp. 524527.

[3] Sa. Dhouib and S. Dhouib, "Optimizing the Trapezoidal Fuzzy Travelling Salesman Problem Through Dhouib-Matrix-TSP1 Method Based on Magnitude Technique", International Journal of Scientific Research in Mathematical and Statistical Sciences, 2021, vol. 8, Issue 2 pp. 1-4.

[4] L.A. Zadeh, "Fuzzy sets", Information and Control, 1965, ,vol.8, pp. 338-353.

[5] L.A. Zadeh, "Fuzzy sets as a basis for a theory of Possibility", Fuzzy sets and Systems, 1978, vol. 1, pp. 3-28.

[6] A. Aslan, "A Memetic Random Key Algorithm for the Balanced Travelling Salesman Problem", Metaheuristics for Combinatorial Optimization, 2021, pp.16-22.

[7] Y. Saji and M. Barkatou, "A discrete bat algorithm based on Lévy flights for Euclidean traveling salesman problem", Expert Systems with Applications, 2021, vol.172, pp.114639.
[8] A. A. Mehmet, C. B. Kalayci, "A Variable Neighborhood Search Algorithm for Cost-Balanced Travelling Salesman Problem", Metaheuristics for Combinatorial Optimization, 2021, pp. 2336.

[9] S. Chowdhury, M. Marufuzzaman, H Tunc, L. Bian and W. Bullington "A modified Ant Colony Optimization algorithm to solve a dynamic traveling salesman problem A case study with drones for wildlife surveillance", Journal of Computational Design and Engineering, 2019, vol. 6 , issue 3, pp. 368-386.

[10] P. K. P. Ghadle and P. A. Pathade, "Solving Transportation Problem with Generalized Hexagonal and Generalized Octagonal Fuzzy Numbers by Ranking Method", Global Journal of Pure and Applied Mathematics, 2017, vol. 13, number 9, pp. 6367-6376.

[11] C. Changdar, G.S.Mahapatra and R. K. Pal, "An efficient genetic algorithm for multi-objective solid travelling salesman problem under fuzziness", Swarm and Evolutionary Computation, 2014, vol. 15, pp. 27 37.

[12] Y. Liao, D. Yau and C. Chen, "Evolutionary algorithm to traveling salesman problems", Computers \& Mathematics with Applications, 2012, vol. 64, issue 5, pp. 788-797.

[13] Z. Wang, J. Guo, M. Zheng and Y. Wang, "Uncertain multiobjective traveling salesman problem", European Journal of Operational Research, 2015, vol. 241, issue 2, pp. 478-489.

[14] A. Gupt and A. Kumar, "A new method for solving linear multiobjective transportation problems with fuzzy parameters", Applied Mathematical Modelling, 2012, vol 36, issue 4, pp. 1421-1430.

[15] A. Robati, G. A. Barani, H. N. A. Pour and M. J. Fadaee, "Balanced fuzzy particle swarm optimization", Applied Mathematical Modelling, 2012, vol. 36, issue 5, pp. 2169-2177.

[16] J. Majumdar and A. K. Bhunia, "Genetic algorithm for asymmetric traveling salesman problem with imprecise travel times", Journal of Computational and Applied Mathematics, 2011, vol. 235, issue 9, pp. 3063-3078. 